ACTA UNIVERSITATIS LODZIENSIS

Folia Litteraria Romanica 15, 2020

https://doi.org/10.18778/1505-9065.15.13

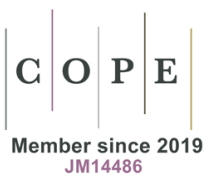

Anna Opiela-Mrozik

Université de Varsovie

iD ORCID ID : 0000-0002-4349-0631

am.opiela@uw.edu.pl

\title{
Les faces du mépris chez Villiers de l’Isle-Adam
}

\begin{abstract}
RÉSUMÉ
L'article présente la genèse et les caractéristiques du mépris, ce sentiment et attitude complétés de dédain, qui constitue l'une des notions clés dans l'esthétique de Villiers de l'Isle-Adam. Le mépris, mis à l'aide de l'ironie au double service du " rêveur et railleur » en quête de la gloire, reste particulièrement protéiforme et révélateur de la philosophie villiérienne. À côté du mépris idéologique visant les valeurs matérialistes de la société bourgeoise qui détruit l'idéal, l'œuvre de Villiers fait voir le mépris scientifique dirigé contre l'idée du progrès, et le mépris journalistique, inspiré par les expériences de l'écrivain condamné à l'échec. Au niveau psychologique, le dédain peut être feint et le mépris présupposé, mais aussi inhérent à l'amour, ce qui conduit à un acte d'auto-dévalorisation. L'article analyse la lutte entre ces deux passions qui se déroule au moyen du langage et des gestes.
\end{abstract}

MOTS-CLÉS - mépris, dédain, ironie, bourgeois, science, gloire, amour, Villiers de l'Isle-Adam

“The Different Faces of Contempt in the Villiers de l'Isle-Adam's Euvre"

\section{SUMMARY}

This article presents the origins and characteristics of contempt in the Villiers de l'Isle-Adam's work. This feeling, complemented by disdain, is one of the key-terms in the aesthetics of this writer in search of glory. He used it as a dreamer and a scoffer as well; this term takes different forms and reveals Villiers' philosophy. Next to the ideological contempt aimed at the materialistic values of bourgeois society which destroy the ideal, the Villiers' œuvre also shows the scientific contempt aimed at the idea of progress and the journalistic contempt which has its origin in his experience of a writer who is doomed to failure. On the psychological level, the contempt can be pretended or assumed but it can also coexist with love, which leads to self-depreciation. This article analyses the struggle between two passions which proceeds by words and gestures.

KEYWORDS - contempt, disdain, irony, bourgeois, science, glory, love, Villiers de l'Isle-Adam 
On connaît bien la dédicace de L'Ève future dans laquelle Villiers a relié les rêveurs aux railleurs, en résumant ainsi les deux courants de sa création. Dans sa quête de l'idéalisme, c'est au moyen du mépris hautain qu'il dévoilait les platitudes de l'existence et les limites du progrès et de la science. Par ailleurs, toute l'histoire de Villiers de l'Isle-Adam a été placée sous le signe d'un écart entre le rêve de gloire et la réalité d'un échec quasi constant, auquel s'ajoutaient les difficultés d'une vie de misère que devait mener cet aristocrate issu d'une ancienne famille bretonne. Tiraillé en permanence entre les extrêmes, Villiers a été contraint de subir le sort d'un écrivain ambitieux mais incompris par le public, ce qui a d'autant plus favorisé l'adoption d'une attitude méprisante envers les hommes et le monde qui l'entourait. Refusant de se résigner à une destinée paradoxale, dont témoignait sa lutte quotidienne pour survivre, mais surtout la foi inébranlable en la primauté de l'esprit sur la matière, Villiers avait selon Verlaine un « orgueil immense, justifié $»^{1}$, tandis que son œuvre exprimait, au dire de Remy de Gourmont, « le mépris indigné $»^{2}$.

Cependant, pour reprendre la définition du mépris qui désigne le «peu de prix, peu d'estime que l'on attache à une personne ou à une chose, idée désavantageuse que l'on conçoit ou que l'on affecte $»^{3}$, il semble que, dans le cas de Villiers, il s'agisse, plus précisément, de dédain qui signifie le « mépris orgueilleux ». En effet, contrairement au mépris qui ne vise que l'objet sous-estimé en raison des qualités qui lui sont propres, le dédain « vient de la haute idée qu'une personne a d'elle-même ; il ne suppose pas que l'objet dédaigné soit mauvais en lui-même, mais seulement qu'on le juge indigne de son attention $»^{4}$. D'ailleurs, comme le souligne une philosophe et anthropologue britannique, Tiffany Watt Smith, le mépris s'identifie toujours à une émotion aristocratique qui pénètre l'homme d'un sentiment de supériorité derrière lequel se dissimule la moquerie ou le dégoût ${ }^{5}$. C'est pourquoi, quelle que soit la forme de son attitude, un dédaigneux traite l'autre avec une indifférence voilée de plaisanterie.

Il n'y a qu'un pas jusqu'à la raillerie et à la dérision, ces deux « cousins $»^{6}$ du mépris, que Villiers prend comme armes dans son combat littéraire contre la réalité décevante marquée par la pensée « positive » et les valeurs matérialistes. Ainsi, exploitant largement les ressources de l'ironie, Villiers place-t-il toute son esthétique sous le signe de vengeance dont le projet littéraire se profile à travers sa

1 P. Verlaine, Les Poètes maudits, in Euvres en prose complètes, éd. J. Borel, Paris, Gallimard, 1972, p. 678.

2 R. de Gourmont, Sixtine. Roman de la vie cérébrale, Paris, Union Générale d'Éditions, 1982, p. 110.

3 Grand dictionnaire universel du XIX $X^{e}$ siècle, éd. P. Larousse, Paris, 1874, vol. XI, p. 47.

4 Ibid., 1870, vol. VI, p. 270.

5 Cf. T. Watt Smith, Księga ludzkich uczuć [Le Livre des sentiments humains], trad. J. Konieczny, Warszawa, Wydawnictwo WAB, 2015, p. 195-196.

${ }^{6}$ Ibid., p. 196. 
correspondance. « Je travaille à nous venger $»^{7}$, écrit-il à Mallarmé en soulignant son affinité avec cet aristocrate d'esprit s'adressant à une élite intellectuelle. Pour Villiers, l'écriture relève d'un acte de justice conjugué au désir de gloire que le sentiment d'indignation face à la dégradation du public ne peut que renforcer.

Cela traduit la récurrence de la notion de « mépris » qui, complétée et relayée par celle de « dédain », constitue l'un des mots clés dans le vocabulaire villiérien. D'autant plus qu'il se prête à l'ambiguïté caractéristique pour l'attitude villiérienne reliant « la colère bouillante du poète exterminateur et le froid mépris de l'ironiste impassible, le tout mêlé à l'humour pince-sans-rire de l'artiste pourfendeur de la stupidité bourgeoise ${ }^{8}$. Placé entre l'ironie qui contribue à la "cruauté » émanant de l'écriture, et l'orgueil qui permet à l'écrivain de poursuivre le rêve d'une aristocratie de lettres, le mépris occupe une place importante dans l'esthétique villiérienne. Mais, qui plus est, il reste une catégorie psychologique qui, à travers les émotions et les réactions des personnages et du narrateur, complète la position dédaigneuse adoptée par Villiers et dévoile l'ampleur de son ambition esthétique. Comme les origines de l'attitude méprisante de Villiers renvoient à sa biographie, nous nous proposons de rappeler quelques points importants de l'histoire personnelle de l'écrivain pour ensuite, dans quelques-uns de ses textes, relever les divers faces du mépris, et notamment sa psychologie.

\section{Comment devient-on dédaigneux ?}

Il est important de mentionner que c'est dans l'enfance du futur écrivain qu'il faudrait chercher la genèse de l'attitude qui, d'un côté, empêchait de réussir dans la vie sociale, mais de l'autre, assurait de riches ressources littéraires. Villiers est né dans une famille de nobles appauvris qui cultivaient le mythe de leur passé glorieux. Se croyant descendant des chevaliers médiévaux, le jeune Villiers n'a pas pu échapper à son destin d'être exceptionnel, d'autant plus que son père, un inlassable chercheur d'or, développait en lui le rêve d'un trésor fabuleux enfoui par leurs ancêtres dans un endroit mystérieux. En tant que fils unique et enfant prodige chéri par ses proches, il ne devait que triompher, confirmant ainsi l'honneur de son nom. Adolescent solitaire, il a choisi de se distinguer des autres afin de cultiver son imagination débordante. Selon les suggestions notées à l'époque par sa cousine, il aurait évité de jouer avec les enfants, comme si «ça l'humiliait »'. Le futur génie

7 Correspondance générale de Villiers de l'Isle-Adam, éd. J. Bollery, Paris, Mercure de France, 1962, t. I, p. 99.

8 J. Noiray, «La littérature comme vengeance : l'esthétique de Villiers de l'Isle-Adam d'après sa correspondance ", in L'Esthétique dans les correspondances d'écrivains et de musiciens (XIX ${ }^{e}-X X^{e}$ siècles), éd. A. Michel, Paris, PUPS, 2001, p. 55.

9 Cf. J.-P. Bourre, Villiers de l'Isle-Adam. Splendeur et misère, Paris, Les Belles Lettres, 2002, p. 29. 
préférait développer ses talents et sa sensibilité artistiques, tout en prenant le goût de la mystification.

Ce fut d'ailleurs grâce à ses discours enflammés, espèces de soliloques vertigineux prononcés dans des cafés parisiens, qu'il se fit connaître auprès de la bohème artistique après son arrivée à la capitale dans l'intention bien précise d'y faire une carrière littéraire. Toujours double dans ses rôles improvisés, considéré soit comme un génie, soit comme un être étrange et agaçant, Villiers s'est pourtant, au cours des années, constamment heurté au refus du public. La vanité blessée, conjuguée aux idées de Baudelaire qu'il admirait ${ }^{10}$, ne pouvaient qu'engendrer une attitude de distance pleine d'ironie et de dédain pour la médiocrité. Villiers a finalement adopté sa position de " poète maudit » qui, même après la parution de ses Contes cruels (1883), n’a pas reçu ce qui lui était dû : « Bien que Villiers soit déjà TRÈS GLORIEUX, et que son nom parte, destiné au plus profond retentissement, pour une postérité sans fin, néanmoins nous le classons parmi les Poètes maudits, PARCE Qu'IL N'EST PAS ASSEZ GLORIEUX en ces temps qui devraient être à ses pieds $\gg^{11}$, explique Verlaine. Mallarmé rappelle la volonté de Villiers qui ambitionnait de « régner », selon la conviction que pour compléter l'illustration de sa race, il aurait été nécessaire d'atteindre à « la seule gloire vraiment noble de nos temps, celle d'un grand écrivain $\gg^{12}$.

Cependant, ayant, dès sa jeunesse, une idée de la gloire gardée au fond de luimême, il a dû subir un décalage douloureux entre ce qui était et ce qui, à son avis, devait être. Cette incompatibilité entre l'intérieur et l'extérieur, que Villiers essayait en vain de surmonter, l'a inévitablement conduit à poser un voile d'indifférence dédaigneuse sur presque tous les aspects de la vie décrits dans ses textes. Si l'on considère la situation de Villiers à partir des années 1860, qui était celle d'un homme dépouillé de tout, on y voit l'histoire de l'artiste absolu, du poète de l'idéal qui a choisi de «s'épuiser à vivre en rêvant $»^{13}$. Les réflexions de Mallarmé suggèrent que Villiers illustre la générosité de l'artiste poussée à l'extrême au nom de l'idéal, un artiste qui « s'est donné, s'est produit lui-même tout entier dans son époque $»^{14}$. Or, Thomas Conrad souligne le caractère dialogique de la notion d'idéal, dont découle le rapprochement entre « le mystère de l'idéal » et « la mystification du bourgeois » : c'est « contre une certaine idée du réel, contre un certain genre

\footnotetext{
${ }^{10}$ Ryszard Engelking, auteur de la nouvelle traduction polonaise de L'Ève future (2015), souligne l'importance de la rencontre de Villiers avec Baudelaire à qui il a emprunté le mépris de la médiocrité et des règles, l'ironie violente et la conscience de son individualisme. $C f$. R. Engelking, „Marzyciel szyderca” [«Rêveur railleur »], Literatura na świecie, 2001, nº 1, p. 275.

${ }^{11}$ P. Verlaine, Les Poètes maudits, op. cit., p. 679.

${ }^{12}$ Cf. S. Mallarmé, Villiers de l'Isle-Adam, in Euvres complètes, éd. B. Marchal, Paris, Gallimard, 2003 , t. II, p. 31.

13 J.-P. Bourre, op. cit., p. 146.

${ }^{14}$ S. Meitinger, « Mallarmé, poète et histrion », Romantisme, 1987, nº 55, p. 99.
} 
du lecteur », incapable de saisir le message, qu'on revendique cet idéal ${ }^{15}$. L'ironie, qui chez Villiers sert largement à exprimer le mépris, apparaît donc comme un lien nécessaire entre le réel observé et l'idéal recherché. La satire et le lyrisme, ces deux dimensions de l'écriture villiérienne, en apparence contradictoires, se rejoignent et se complètent, ce qui, paradoxalement, justifie, sur le plan esthétique, le mépris adopté par le narrateur. Par ailleurs, c'est également Mallarmé qui insistait sur le décalage existant entre Villiers et ses contemporains incapables de saisir la modernité de son écriture à deux versants, " selon les modes en secret correspondant du Rêve et du Rire $»^{16}$. C'est dans ce lien mystérieux que se cache la vérité sur l'homme et sur le monde, et c'est là que réside « la plus parfaite symétrie d'âme qui fut jamais » ${ }^{17}$. Quelque complexe que soit sa genèse dans l'écriture villiérienne, le mépris apparaît donc comme un des éléments qui assurent la cohésion de la philosophie de l'écrivain.

\section{2. À bas les bourgeois !}

La diversité des objets que vise le mépris villiérien exige de procéder par une sorte de classement, ce qui permettrait de saisir ses aspects multiples. Il nous paraît nécessaire de commencer par un mépris idéologique, proche de celui de Flaubert. En effet, c'est en recherchant son propre style, détaché des modèles romantiques, que Villiers a créé le personnage de Tribulat Bonhomet, un bourgeois typique qui, dans sa sottise et sa cruauté aveugle, dépasse même le pharmacien flaubertien. Comme l'écrivain l'avoue dans une lettre à Mallarmé, son personnage grotesque devait s'inscrire dans la lignée des portraits satiriques du passé et marquer la violence de son ironie démystificatrice :

Le fait est que je ferai du bourgeois, [...] ce que Voltaire a fait des 'cléricaux', Rousseau des gentilshommes et Molière des médecins. Il paraît que j'ai une puissance du grotesque dont je ne me doutais pas. Enfin nous rirons un peu. On m'a dit que Daumier les flattait servilement en comparaison. Et naturellement, moi j'ai l'air de les aimer et de les porter aux nues, en les tuant comme des coqs ${ }^{18}$.

Bonhomet, qui se définit comme un docteur, philanthrope et homme du monde, réunit donc tous les vices de sa classe et de son époque, en devenant ainsi l'archétype suprême du bourgeois. Ce fameux « tueur de cygnes », le « preux moderne » qui

${ }^{15}$ Th. Conrad, «Le laboratoire de Villiers de l'Isle-Adam : manières d'écrire l'idéal », p. 4, in Ce qu'idéal veut dire : définitions et usages de l'idéalisme au XIXe siècle, éd. C. Echiffre et al., 2015 ; URL : http://etudes-romantiques.ish-lyon.cnrs.fr/idealisme.html ; consulté le 8.01.2019.

${ }^{16}$ S. Mallarmé, Villiers de l'Isle-Adam, op. cit., p. 44.

${ }^{17}$ Ibid., p. 44.

${ }^{18}$ Correspondance générale de Villiers de l'Isle-Adam, op. cit., t. I, p. 99. 
sacrifie sans scrupules les " oiseaux-poètes $»^{19}$ au nom d'une sensation absurde, devient une projection symbolique de tout ce que Villiers haïssait chez les bourgeois : le matérialisme poussé à l'extrême, l'auto-satisfaction fondée sur la sottise sans bornes, le culte aveugle du progrès et de l'argent. Plus encore : confronté aux êtres spirituels, il acquiert une dimension diabolique qu'il ne suffit pas de mépriser, mais avec laquelle on est obligé de lutter.

D'autant plus que chacun risque de s'immerger dans la trivialité de la vie. Villiers lui-même ne s'en croit pas exempt en avouant : " Je hais le bourgeois, mais le bourgeois dont je parle, j'en trouve autant sous la blouse que sous les noms les plus illustres du monde $»^{20}$. Dans plusieurs de ses contes Villiers entreprend de dénoncer les composantes de l'esprit bourgeois, en mettant ainsi son mépris au service de la satire. Sans épargner le lecteur, l'écrivain décrit alors le carnage des deux groupes des bourgeois, habitants des contrées voisines, qui se prenant les uns les autres pour des voleurs, déchaînent leur férocité animale et s'entre-tuent, du fait que «l'instinct de la conservation de leurs vies et de leur argent les aveuglait»" ${ }^{21} \mathrm{Au}$ moyen d'une parodie du style héroïque complété du motif médiéval du « trompeur trompé », Villiers réussit à obtenir un effet comique qui incite le lecteur à adopter le point de vue du narrateur et partager son mépris à l'égard des petits bourgeois et de leur médiocrité. Cela se produit également dans Impatience de la foule où Villiers ironiste condamne la sottise bourgeoise qui ne tarde pas à se transformer en violence précipitée et injuste envers le messager. En revanche, l'attachement aux intérêts matériels donne lieu à une scène (Virginie et Paul), dont le poids intertextuel, ainsi que le décor apparemment romantique, ne font qu'amplifier l'ironie de Villiers : dans l'amour bourgeois ce n'est que l'argent qui compte ${ }^{22}$. Il en est de même avec les relations humaines en général, ce dont témoigne l'attitude des convives du Plus Beau Dîner du monde. Ayant découvert le menu dudit « plus beau dîner du monde » de l'année précédente, donné par $\mathrm{M}^{\mathrm{e}}$ Percenoix, les invités se sont réunis chez son rival dans « l'impression du profond mépris » pour leur hôte ${ }^{23}$. Une pièce de vingt francs cachée sous l'assiette de chacun permet pourtant de remplacer cette

19 A. Villiers de l'Isle-Adam, Tueur de cygnes, in Euvres complètes, éd. A. Raitt et P.-G. Castex, Paris, Gallimard, 1986, t. II, p. 135.

${ }^{20}$ Idem, Fragments divers, in Euvres complètes, op. cit., t. II, p. 1001.

${ }^{21}$ Idem, Les Brigands, in CEuvres complètes, op. cit., 1986, t. I, p. 678.

${ }^{22}$ Il est intéressant de remarquer que la conclusion du conte met le lecteur dans la difficulté. Certes, le récit de Villiers est conçu sur le mode de la parodie du roman de Bernardin de Saint-Pierre, Paul et Virginie, qui fournit un modèle de l'amour juvénile. Cependant, la conclusion du conte paraît ambiguë en raison d'une remarque mise en italique : «Soyez bénis, enfants, dans votre extase ! vous dont l'âme est simple comme la fleur, vous dont les paroles, évoquant d'autres souvenirs à peu près pareils à ce premier rendez-vous, font verser de douces larmes à un passant !» (Virginie et Paul, in CEuvres complètes, op. cit., 1986, t. I, p. 606). Les mots « à peu près » mettent en doute toute l'interprétation du texte : on peut se demander si Villiers continue à se moquer des adolescents modernes ou s'il suggère l'omniprésence de l'esprit bourgeois auquel personne ne peut échapper.

${ }^{23}$ Idem, Le Plus Beau Dîner du monde, in Cuvres complètes, op. cit., 1986, t. I, p. 654. 
impression par une autre, indéfinissable, qu'on assiste à un dîner plus beau que le premier. En faisant un clin d'œil au lecteur, Villiers se plaît ainsi à déployer sa critique des bourgeois de façon à peindre leurs portraits-charges.

Le bourgeois comme un objet de dédain se retrouve également dans le conte intitulé L'Amour sublime qui met en scène un mari jaloux d'une liaison spirituelle entamée par sa femme avec un cousin, son âme sœur. Ce qui est intéressant, c'est que le dédain crée une espèce de cercle vicieux où chacun des personnages est enfermé. Le mari, un autre bourgeois typique, traite sa femme « atteinte d'âme $»^{24}$ avec une indulgence réfléchie et se croit supérieur au cousin de celle-ci, non moins spirituel qu'elle et dédaignant les choses terrestres, tandis que lui-même, il reste un objet de dérision complète de la part de Villiers qui met en œuvre les ressources de l'ironie afin d'en donner un portrait exact, digne de celui de Bonhomet. Qui plus est, selon une logique utilitaire, le comportement de sa femme honnête et charitable prend pour lui un aspect de flatterie, de sorte que « ce vice de conformation ne lui semblait pas absolument rédhibitoire $»^{25}$. Étrangère à son entourage et traitée avec une bonne dose de moquerie voire de mépris propre aux esprits positifs qui « la déclaraient 'supérieure' avec un demi-sourire qui servait la transition pour parler de choses plus gaies $»^{26}$, elle reste un objet d'admiration de la part du narrateur villiérien qui la décrit en tant qu'un être d'au-delà, simple et spirituelle comme une sainte.

Cependant, après l'arrivée du jeune homme et l'amour idéal qui se noue entre les deux êtres rapprochés par leur nature, c'est le progrès du mépris qui conduit le mari à faire appel à un commissaire de police pour l'aider à tendre un piège aux amants. Par ailleurs, Villiers témoigne de la sympathie à l'égard du nouveau venu, le jeune Bénédict, en approuvant son indifférence envers les affaires du monde et la décision de ne vivre que dans son intérieur, étant donné que le « farouche conflit d'intérêts [...] étouffe d'avance, sous le ridicule et le dédain, tout effort tenté vers quoi que ce soit d'élevé, de désintéressé, de digne d'être " ${ }^{27}$. Le bourgeois possède ce que Bénédict néglige : c'est un homme ayant « le sentiment de sa rationnelle supériorité $»^{28}$, qui se refuse à prendre au sérieux les jeux des enfants amoureux.

Mais à mesure que continue cette relation irréprochable, dont le sens profond échappe au bourgeois réaliste à outrance, ses émotions évoluent, si bien que la neutralité fondée sur la " dédaigneuse pitié qu'il ressentait $»^{29}$, diminue son amour pour la femme, qu'il finit par humilier. Telle est donc la transformation psychologique qui s'est opérée en lui : le sentiment de mépris, tout rationnel qu'il puisse paraître, a été remplacé par celui de dégoût, suivi immédiatement du désir d'humilier les prétendus coupables. C'est une « dédaigneuse aversion pour

\footnotetext{
${ }^{24}$ Idem, L'Amour sublime, in Cuvres complètes, op. cit., t. II, p. 712.

${ }^{25}$ Ibid., p. 714.

${ }^{26}$ Ibid., p. 713.

${ }^{27}$ Ibid., p. 716.

${ }^{28}$ Ibid., p. 718.

${ }^{29}$ Ibid., p. 719.
} 
cette malheureuse insensée $»^{30}$ qui amène finalement le pauvre mari à concevoir l'idée de divorce comme l'unique issue de la situation insupportable. En effet, du point de vue de la psychologie, lorsqu'on éprouve du dégoût, c'est l'objet de répulsion qui se présente le plus nettement à notre conscience. Son caractère dégoûtant apparaît pour le sujet comme une affection qui couvre cet objet d'une couche de mucus ${ }^{31}$. Mais la conclusion de Villiers ne laisse pas de doute : ridiculisé par sa femme, dont l'amour platonique dépasse sa capacité de compréhension limitée à la vulgaire réalité du désir, le mari, ayant subi un choc, risque de perdre sa raison jusque-là inébranlable. À la vue du couple amoureux récitant ensemble leur prière du soir, il se trouve impuissant face à la force de l'idéal qu'il ne cesse de refuser. C'est ainsi qu'il devient une victime exemplaire de la cruauté villiérienne. Son dédain de la religion et de l'âme en général se retourne contre lui-même, ce dont témoigne la réaction méprisante du commissaire de police : "'Pauvre ami ! Pas MÊME... trompé !...'» $\rangle^{32}$. La vengeance anti-bourgeoise de Villiers, aussi efficace que choquante, va au-delà même du mépris.

\section{Regard oblique sur la science et sur l'art}

Dans son texte sur Villiers, Mallarmé souligne le défi total que celui-ci a lancé à la médiocrité, mais aussi « l'éperdu combat que le querelleur mena contre toute infatuation moderne, qu'elle s'appelât industrie, progrès, même Science $»^{33}$. Le mépris dit scientifique révèle une résistance villiérienne à la modernité, qui prend forme d'une esthétique, dont témoigne L'Ève future. Malgré la fascination de Villiers pour le mystère de la science et ses potentialités associées au sublime, le roman dénonce les limites du savoir : non seulement par l'échec final de l'entreprise d'Edison, mais aussi par l'accumulation et la description de détails et d'opérations techniques, ce qui, au lieu d'expliquer la science, contribue à sa décomposition. D'ailleurs, la science ne joue qu'un rôle auxiliaire dans la création du rêve. Si Edison se permet de développer devant Lord Ewald ses discours pseudo-scientifiques, il n'oublie pourtant pas les réticences de son ami en lui demandant, par exemple, de n'accorder aux oiseaux électriques qu'une « dédaigneuse attention ${ }^{34}$.

La défense de l'idéal au moyen d'une ironie acerbe, insérée dans un discours journalistique, s'effectue chez Villiers à travers des contes consacrés aux inventions techniques, aussi modernes qu'absurdes. Voici donc une machine qui remplacerait

\footnotetext{
${ }^{30}$ Ibid., p. 720.

${ }^{31}$ Cf. S. Tomkins, „Wstyd-upokorzenie a pogarda-wstręt: natura reakcji” [" Honte-humiliation et mépris-répulsion : la nature des réactions »], trad. B. Szumański et W. Szwebs, Teksty Drugie, 2016, no 4, p. 171.

${ }^{32}$ A. Villiers de l'Isle-Adam, L'Amour sublime, op. cit., p. 722.

${ }^{33}$ S. Mallarmé, Villiers de l'Isle-Adam, op. cit., p. 26.

${ }^{34}$ A. Villiers de l'Isle-Adam, L'Ève future, in CEuvres complètes, op. cit., t. I, p. 871.
} 
la claque traditionnelle et, soutenue, en cas de nécessité, par une vingtaine d'androïdes exprimant leur « mépris profond » pour l'auteur dramatique, guiderait la condamnation de la pièce par le public ${ }^{35}$. De même, cet appareil servirait à produire la « gloire »d'un auteur recommandable selon le goût du public bourgeois ${ }^{36}$. Le succès étant une affaire commerciale, le ciel peut être « considéré au point de vue industriel et sérieux $\gg{ }^{37}$ et devenir, pour sa part, un immense panneau publicitaire, en vue de rendre des services « à la société et au Progrès $\rangle^{38}$. Non moins ironique paraît la description enthousiaste du fonctionnement de l'appareil pour l'analyse chimique du dernier soupir dont l'usage devrait calmer voire abolir des émotions de douleur profonde accompagnant la perte de ses proches. Dans un style vif qui fait penser à une annonce publicitaire, Villiers traduit, sous cette forme provocatrice, son dédain du positivisme et du scientisme, deux ennemis de l'homme moderne. La série des inventions du Progrès triomphant se clôt par les effets du traitement du docteur Tristan dont le prénom fait inévitablement penser à Wagner ${ }^{39}$. Cependant, la cure proposée par ce pseudo-médecin consiste à faire perdre au patient son ouïe intérieure ou, autrement dit, à le priver de remords, de doutes, d'ambitions, son esprit étant « bien délivré de toutes ces Voix vaines et confuses » qui lui causaient des « bourdonnements de gloire, d'honneur, de courage ». « Le vieil Idéal assassiné », c'est « un mépris éclairé de toutes les offenses $»^{40}$ qui remplace des inquiétudes existentielles. L'homme assourdi par la science devient, conformément à la définition du mépris, indifférent aux reproches et conscient de sa supériorité.

Exprimer son dédain de façon raffinée, en faisant croire qu'il s'agit de l'admiration ou même de flatteries, tel est le premier objectif des mystifications villiériennes visant aussi bien l'univers des artistes. À en croire Remy de Gourmont, c'était une attitude adoptée par Villiers lui-même dans ses relations avec les éditeurs et directeurs des revues où il publiait ses textes :

J'allai avec lui au Gil-Blas. Nous voulions offrir à Guérin [...] un roman que je venais de finir. Villiers recommande le manuscrit du ton le plus équivoque, assurant que c'était

${ }^{35}$ Idem, La Machine à Gloire, in Cuvres complètes, op. cit., t. I, p. 593.

${ }^{36}$ Le discours ironique de Villiers ne cesse pourtant pas de se retourner contre lui-même selon la logique de l'ambiguïté : la claque automatique contiendrait donc sa propre négation au point de crier « à bas la claque !» ou de provoquer des discussions entre les spectateurs sur l'art pour l'art!

${ }^{37}$ Idem, L'Affichage céleste, in Cuvres complètes, op. cit., t. I, p. 577.

${ }^{38}$ Ibid., p. 579.

${ }^{39}$ Il ne fait pas de doute que Villiers était un grand admirateur de Wagner et de sa musique. C'est pourquoi, cette allusion au compositeur semble relever d'une espèce de jeu qui dévoile une tension villiérienne au plaisir pervers du renversement des valeurs ou bien à l'ambigu. Tel paraît être également le sens qui se dégage du conte dédié à Wagner, Le Secret de l'ancienne musique, où le ridicule touche non seulement le vieux maître d'un ancien instrument mais aussi les musiciens de l'Académie nationale de Musique qui s'apprêtent à exécuter l'ouvrage d'un certain compositeur allemand (il s'agit, évidemment, de Wagner).

${ }^{40}$ A. Villiers de l'Isle-Adam, Le Traitement du docteur Tristan, in Euvres complètes, op. cit., t. I, p. 733. 
mondain, sensuel, pervers, plein de soupers, de fêtes et de courtisanes, ce qui était bien loin de la vérité. Il affectait d'ailleurs devant ces hommes-là plus singulière attitude, les accablant de saluts, de compliments, se glissant, en humble collaborateur, heureux d'évoluer parmi tant de maîtres. C'était sa manière de mépriser ${ }^{41}$.

Dans Deux augures, en souvenir de ses propres expériences, l'écrivain met en scène un directeur du journal à qui il oppose un littérateur débutant. Serviteur de la médiocrité bourgeoise, le Journaliste, avec un geste éloquent, découvre la supercherie du Poète, apparemment privé de talent : «Au bout de trois minutes, le directeur tressaille, puis rejette, avec dédain, les feuilles volantes sur la table $»^{42}$. Suit une longue tirade du Journaliste qui accuse l'inconnu de vouloir humilier le public : « Mais le pire, c'est que vous laissez pressentir dans l'on ne sait quoi de votre phrase que vous cherchez à dissimuler votre intelligence, pour ne pas effaroucher le lecteur $! »^{43}$. Le Journaliste est ici nettement opposé au Poète, de même que la valeur économique de la presse se détache de la valeur littéraire. Par la réaction du directeur, Villiers attire l'attention sur la supériorité avec laquelle le journalisme, devenu dès lors une industrie culturelle, traite les hommes de lettres. Comme le remarque Landry Liébart, le fondement de la critique villiérienne visant le journalisme réside dans la réflexion esthétique sur la littérarité même du langage qui, par sa « communicabilité » et sa fonction poétique rompt avec les fonctions de « communication » et de référentialité du discours de presse $\mathrm{e}^{44}$. Néanmoins, la réaction du jeune littérateur aux reproches du Journaliste brouille les pistes interprétatives du récit : au lieu de défendre la littérature, il se met à revendiquer les mêmes valeurs de médiocrité qu'expose le directeur, en partageant sa position de cynique ${ }^{45}$. Le dédain du Journaliste se retourne contre lui-même au moment où il est attaqué avec sa propre arme.

Adhérant aux idées de Mallarmé, Villiers s'opposait à l'hégémonie du journalisme qui favorisait, par exemple, le roman populaire, au détriment d'une création plus ambitieuse. S'il n'avait pas d'estime pour des auteurs médiocres, il le manifestait au moyen d'improvisations ironiques, comme celle dont se souvient Remy de Gourmont :

${ }^{41}$ R. de Gourmont, « Un carnet de notes sur Villiers de l'Isle-Adam », L'Ermitage, 15 avril 1906, p. 224. Force est de remarquer que malgré ce dédain ironique visant la presse, c'est elle qui a, paradoxalement, favorisé l'œuvre de Villiers. Sa carrière littéraire était inséparable de son activité journalistique : il publiait régulièrement ses récits en revue, en attendant de les réunir dans le recueil des Contes cruels paru en 1883. Avec une certaine notoriété qui a suivi la parution du volume vient également la possibilité de se faire publier dans quelques principales revues (Gil Blas, Le Figaro, La Vie moderne et autres), dont Villiers ne tarde pas à profiter en publiant $L$ 'Ève future en feuilleton.

42 A. Villiers de l'Isle-Adam, Deux augures, in Euvres complètes, op. cit., t. I, p. 571.

${ }^{43}$ Ibid., p. 572.

${ }^{44} C f$. L. Liébart, «Un exemple d'intersection entre littérature et sociologie : le rapport entre l'écrivain et le journaliste au XIX ${ }^{\mathrm{e}}$ siècle », Equinoxes, printemps/été 2009, no 12, n. p. ; URL : https://www. brown.edu/Research/Equinoxes/journal/Issue\%2012/eqx12_Liebart.html; consulté le 13.01.2019.

${ }^{45}$ Voir à ce propos B. Vibert, «Conte cruel, conte cynique. Villiers, Mirbeau», in Cynismes littéraires, sous la dir. de P. Glaudes, J.-F. Louette, Paris, Classiques Garnier, 2018, p. 160-161. 
Parfois, quand il méprisait beaucoup un écrivain, un poète à la mode, si son nom venait à être cité, il feignait l'enthousiasme, se lançait dans un fougueux éloge, puis, ayant bien joui des mines consternées de son auditoire, il éclatait de rire. Il me joua cette comédie, un soir, à moi tout seul, à propos d'un poète, déjà ou alors presque célèbre, et qu'il n'est pas temps de nommer ${ }^{46}$.

Et pourtant, lorsqu'il s'agit de l'échec qu'a connu son drame La Révolte, disparu de l'affiche après cinq représentations, l'ironie cède place à une critique violente de la politique théâtrale par laquelle on méprise et trompe le public, dans le souci de servir le sens commun. L'avant-propos ajouté à la pièce après sa chute donne à Villiers l'occasion non seulement de répondre aux reproches des critiques et de remercier ceux qui ont écrit « ces belles pages dédaigneuses $»^{47} \mathrm{en}$ faveur de La Révolte, mais aussi de défendre ses convictions esthétiques au point de formuler des rêves prophétiques. Dès les premiers mots, Villiers prend une position hautaine, en faisant délibérément preuve, dit-il, « d'un orgueil presque égal aux vanités chétives de ceux-là même qui me le reprocheront $»^{48}$. Malgré sa haine du bourgeois, Villiers ne cesse de croire au réveil prochain de la Foule, ce « juge tardif, mais seul juge $»^{49}$. Car, si l'on réprime tout ce qui dépasse les moyens habituels, on ne fait que traiter le public avec dédain, ce qui entraîne en retour celui du public même. Mais viendra l'heure où le théâtre ne donnera plus de pièces à succès, mais celles qui assureront à leurs auteurs la Gloire ${ }^{50}$. Si La Révolte présente un essai d'affranchissement féminin de la médiocrité et du matérialisme bourgeois, elle constitue également la première tentative de se libérer des « règles déshonorantes $»^{51}$ qu'impose la scène française.

\section{Aimer et (mé)priser}

Amour, mépris, vanité : voici trois termes autour desquels sont conçues les relations amoureuses dans l'univers villiérien. Ce qui relève d'un paradoxe : le mépris peut être inhérent à l'amour, devenir un enjeu de l'amour ou dissimuler un vrai amour selon un renversement des valeurs qui déplace la portée de cette

${ }^{46}$ R. de Gourmont, «Un carnet de notes sur Villiers de l'Isle-Adam », op. cit., p. 225.

${ }^{47}$ A. Villiers de l'Isle-Adam, Avant-propos de La Révolte, in Euvres complètes, op. cit., t. I, p. 378.

${ }^{48}$ Ibid., p. 377.

49 Ibid., p. 383.

${ }^{50}$ Et Villiers de conclure ses propos dans la certitude de sa propre gloire qui apparaît comme consubstantielle à cet écrivain défini par son orgueil même : "Celui qui, en naissant, ne porte pas dans sa poitrine sa propre gloire, ne connaîtra jamais la signification réelle de ce mot» (ibid., p. 383). Verlaine a parfaitement saisi dans cette phrase la nature de Villiers, en constatant que « ces paroles [...] donnent tout Villiers de l'Isle-Adam, l'homme et l'œuvre » (Les Poètes maudits, op. cit., p. 678).

${ }^{51}$ A. Villiers de l'Isle-Adam, Avant-propos de La Révolte, op. cit., p. 382. 
attitude envers les autres. S'y ajoutent également des enjeux littéraires où le mépris est traité en tant que moyen de raillerie ironique qui n'a pour but que de relever le bien-fondé de la quête idéaliste. Tel est, en effet, le message du récit qui ouvre le recueil des Contes cruels, intitulé Les Demoiselles de Bienfilâtre, et qui met en scène deux sœurs prostituées dont la conduite, par plaisanterie villiérienne, est présentée comme exemplaire jusqu'au moment où l'une d'elles commet une erreur inadmissible et tombe amoureuse d'un pauvre étudiant. Cet événement attire du mépris non seulement sur celle qui « tourna mal $»^{52}$, en trahissant ainsi son nom, mais aussi sur sa famille et sur sa sœur « vertueuse », qui « s'apercevait de certaines nuances, humiliantes »: « On lui marquait plus de froideur depuis la nouvelle de la malversation d'Olympe. [...] Pour la vraie délicatesse, un rien fait plus de mal souvent que l'outrage grossier $»^{53}$. C'est dans un café qu'a lieu une scène quasi théâtrale : Henriette adresse une " tirade » à sa sœur ostracisée qui, accablée de honte, ne tarde pas à mourir. L'accent final marque une ironie démystificatrice poussée à l'extrême : à la vue des pièces de monnaie brillant entre les doigts de son amant, Olympe, « la conscience apaisée $\|^{54}$, pousse son dernier soupir. Dans l'univers des valeurs renversées le rachat par l'amour s'avère donc impossible. C'est ainsi que, d'après les paroles attribuées à Catulle Mendès, du conte de Villiers se dégage " un irréconciliable mépris pour les consciences perverties, et l'on ne sait quelle terrible leçon morale $»^{55}$.

La question des reproches et du mépris qui brise l'amitié des deux prostituées (Les Amies de pension), de nouveau pour une cause inséparable de l'amour et de l'argent, réapparaît au début des Nouveaux contes cruels où, dans une formule plus grotesque que satirique, Villiers dénonce, encore une fois, la «prostitution morale » ou l'adoption des valeurs matérialistes ${ }^{56}$. À cet égard, il serait intéressant d'analyser la symbolique que revêt chez Villiers le personnage de la prostituée : une femme méprisable et offrant son amour vénal, n'est jamais traitée par l'auteur comme un être inférieur; au contraire, elle se transforme en celle qui, en tant que sujet pourvu de parole, méprise la société au nom de sa propre dignité et d'un système de valeurs où domine l'amour pur. Jean Decottignies souligne l'importance de la parole dont est douée la prostituée villiérienne, car il s'agit d'une parole « détentrice $[\ldots]$ d'une puissance d'oubli que symbolise la nomadisation de toutes les valeurs reçues $»^{57}$.

Tel est, en effet, le message du discours de Maryelle, une demi-mondaine qui explique au narrateur, son ancien amant, la logique de son nouveau comportement

\footnotetext{
${ }^{52}$ Idem, Les Demoiselles de Bienfilâtre, in Euvres complètes, op. cit., t. I, p. 548.

${ }^{53}$ Ibid., p. 548-549.

${ }^{54}$ Ibid., p. 552.

${ }^{55}$ Cf. J. Decottignies, Villiers le taciturne, Lille, Presses Universitaires de Lille, 1983, p. 84.

${ }^{56} C f$. P. Glaudes et B. Vibert, « Le théâtre du demi-monde dans les contes cruels », Littérature, 2014, $\mathrm{n}^{\circ} 71$, p. 224.

${ }^{57}$ J. Decottignies, op. cit., p. 25.
} 
de femme amoureuse et fidèle à un amant innocent. Ce qui pique la curiosité du narrateur, pour qui l'amour relève de la vanité, c'est la certitude réfléchie de la fidélité chez une femme qui continue pourtant sa vie de cocotte : « un frais rire aux notes d'or et de cristal », ainsi qu'un geste de mépris ( «Et elle haussa dédaigneusement les épaules $\gg^{58}$ ) en réponse aux reproches du narrateur. Telle est la réaction de la demimondaine qui, grâce à son système de valeurs idéalistes, peut accéder « à une forme de rédemption textuelle qui s'exhausse par-delà le rire ou l'ironie $»^{59}$. La dignité d'un personnage idéaliste attribuée à la prostituée se trouve confirmée lorsqu'elle se lance dans « une véritable tirade de l'héroïne villiérienne ${ }^{60}$, plaidant la cause générale des femmes qui, indifférentes à la morale bourgeoise, de même qu'au plaisir des sens, deviennent capables de vivre leur vrai amour. La fin du conte permet de voir le narrateur comme victime de ce dédain d'inspiration amoureuse ${ }^{61}$.

Mais dans le monde villiérien le dédain peut se diriger contre l'amour et n'être qu'un moyen permettant de satisfaire des goûts morbides, ce dont témoigne l'attitude du personnage éponyme du conte Sylvabel. Son idée de l'amour est conjuguée à celle de mérite voire de conquête que, selon son désir quasi sadique, doit réaliser son mari fraîchement épousé. Se rendant compte que sa femme le « dédaigne quelque peu » pour ne pas avoir de caractère, celui-ci, de peur de perdre aussi bien le bonheur que l'honneur en s'auto-dévalorisant, demande conseil à son oncle ${ }^{62}$. Mais la lutte entre dédain et amour ne relève que d'un leurre : les épreuves dont triomphe le mari de Sylvabel se ramènent à un jeu cruel inventé par l'épouse. Même si la conquête parait accomplie, le message du conte reste ambigu et profondément misogyne : ce qu'a demandé Sylvabel, apparemment par mépris, s'oppose ici à l'amour idéaliste conçu par Villiers.

Or, une véritable lutte entre le vrai amour contraint de se dissimuler et le dédain affecté et forcé, avec une mise en scène des plus préparées, se déroule dans le conte Sentimentalisme. Comme le suggère l'ironie présupposée du titre, il importe à un jeune homme amoureux d'une coquette capricieuse de ne pas lui faire deviner son côté " sentimental ", et cela même face à l'annonce inattendue de leur rupture. L'enjeu du dialogue reste la faculté de sentir qui, comme le prétend Mme Émery, disparaît chez les artistes " sans cesse agités d'impressions artificielles ${ }^{63}$. Afin de répondre au reproche provocateur de sa maîtresse, Maximilien a recours au discours scientifique inspiré des données sensualistes, mais dès le moment où

\footnotetext{
58 A. Villiers de l'Isle-Adam, Maryelle, in Euvres complètes, op. cit., t. I, p. 727.

${ }^{59}$ P. Glaudes et B. Vibert, op. cit., p. 224.

${ }^{60}$ Ibid., p. 226.

${ }^{61}$ Évoquons ici une autre figure de la prostituée, Antonie, qui témoigne du mépris à l'égard de ses amants. Elle ne le fait pourtant que par l'exaltation de soi-même, telle une beauté immuable, ou l'expression d'un amour narcissique. Son rire joyeux qui cache les vrais sentiments prend une valeur ironique. $C f$. Villiers de l'Isle-Adam, Antonie, in Euvres complètes, op. cit., t. I, p. 582.

${ }^{62}$ Idem, Sylvabel, in Euvres complètes, op. cit., t. II, p. 368.

${ }^{63}$ Idem, Sentimentalisme, in CEuvres complètes, op. cit., t. I, p. 642.
} 
il apprend la trahison de la femme, il se met à jouer le rôle d'un dandy qui reste indifférent aux émotions : «Si le comte, à ces paroles, devint un peu pâle, l'obscurité protectrice voila cette marque d'émotion; nul frémissement ne décela ce que dut subir son être en cet instant $\rangle^{64}$. Tout au contraire : Maximilien, être encore atteint d'âme, oppose sa supériorité émotionnelle au sentimentalisme bourgeois, ce qui permet de voir en lui un homme qui dédaigne l'amour. L'illusion se dissipe dans la scène finale où le héros se suicide d'un coup de pistolet. Tel est donc son « sentimentalisme » qui a prévalu sur la prétendue indifférence.

Si Maximilien dénonce les banalités du langage dont souffrent les poètes amoureux, elles se placent au premier plan dans l'idée de l'amour taché de mépris que ressent Lord Ewald à l'égard d'Alicia Clary. La source de cette émotion à deux versants réside dans le langage par lequel Alicia se dégrade elle-même et tout ce qui l'entoure, faisant ainsi preuve de " surdité intellectuelle et métaphysique ${ }^{65}$. Si Lord Ewald ne cesse d'admirer sa beauté physique, cette femme se ramène pour lui à une " pure extériorité ${ }^{66}$, incapable d'instaurer un lien spirituel avec l'autre : elle est « d'une candeur cynique, dont je ne puis que dédaigner l'inconscience $»^{67}$, avoue-t-il à Edison. C'est pourquoi, il lui préfère son modèle de marbre, la Vénus Victrix, qui dépasse la Déesse bourgeoise grâce à son silence ${ }^{68}$. Voici un point essentiel qui complète la haine villiérienne de la sottise bourgeoise : le mépris du langage déchu, fondé sur la répétition des clichés et privé de sens profond. Paradoxalement, ce n'est pas l'andréïde, mais Alicia, récitant des lieux communs, qui ressemble davantage à un automate.

Selon la précitée théorie du mépris-répulsion, par le fait d'aimer « une dualité animée » qui l'attire et le repousse, Lord Ewald croit s'abaisser dans ses propres yeux, ce qui est pour lui inadmissible : «Toutefois, je ne suis pas d'une nature de subir longtemps l'attrait (si puissant qu'il soit) de ce que je dédaigne à moitié. L'amour où nul sentiment, nulle intelligence ne se mêle à la sensation me semble offensant envers moi-même $»^{69}$. Telle est l'ultime raison pour laquelle Lord Ewald signe un pacte diabolique avec Edison : le remords d'avoir commis un acte d'autodévalorisation par une relation avec cette femme. C'est ainsi qu'Alicia rejoint Miss Evelyn Habal, allégorie de la vanité de l'amour qui, pour reprendre la vision misogyne d'Edison, ne peut qu'engendrer l'aversion et abaisser les hommes, ce dont

\footnotetext{
${ }^{64}$ Ibid., p. 644.

${ }^{65}$ Cf. A. Le Feuvre, Une Poétique de la récitation : Villiers de l'Isle-Adam, Paris, Honoré Champion, 1999, p. 53-54.

${ }^{66}$ Ibid., p. 33.

${ }^{67}$ A. Villiers de l'Isle-Adam, L'Ève future, op. cit., p. 803.

${ }^{68}$ La supériorité du silence sur la parole et le rire qui sert à traduire la sottise, de même que la place privilégiée du silence et ses plusieurs fonctions dans les textes villiériens ont été analysées par Anne-Simone Dufief ( «'Et c'était un crescendo de silences'. Villiers de l'Isle-Adam », in Silences fin-de-siècle. Hommage à Jean Palacio, sous la dir. d'A. Guyaux, Paris, PUPS, 2008, p. 167-179.

${ }^{69}$ A. Villiers de l'Isle-Adam, L'Ève future, op. cit., p. 816-817.
} 
témoigne l'histoire d'Anderson. Lorsqu'il a été délaissé par son amante, le méprisaversion à l'égard de soi-même a été accompagné chez lui du sentiment de «hontehumiliation $»^{70}$. La honte étant l'une des plus importantes motivations, elle a fini par conduire Anderson au suicide, « lorsqu'il se vit ainsi vieilli, désorganisé, amoindri, mésestimé et seul $»^{71}$.

Dans la philosophie villiérienne, le mépris qui nuirait à l'amour véritable est à éviter, même au prix de la solitude, ce que suggère la conclusion du conte L'Inсоппие. Une femme entrevue lors d'une représentation à l'Opéra et abordée au mépris de toute convenance, se révèle être un amour idéal pour le jeune comte Félicien de la Vierge, qui n'est pas sans rappeler Villiers lui-même. Mais la jeune femme, telle la passante baudelairienne, se refuse à l'amour de Félicien en raison de sa surdité et du mépris supposé qui s'ensuivrait et dégraderait leur union, la pensée de l'autre se révélant dans la musique même de ses paroles. Et l'Inconnue d'exprimer son malheur : " cette seule réalité, enfin, je ne la connaîtrai jamais ! Non !... Cette musique ineffable, cachée dans la voix d'un amant, ce murmure aux inflexions inouïes, qui enveloppe et fait pâlir, je serais condamnée à ne pas l'entendre !... $»^{72}$. L'infirmité risquerait donc d'engendrer « le douloureux mépris [...] pour leur sorte d'amour $\gg^{73}$ que l'Amour ne pardonnerait pas.

Autant d'aspects de la vie et de l'amour, autant de faces du mépris qui, à travers un regard ironique, dévoile les convictions de Villiers et donne la clé de l'interprétation visée par l'écrivain, malgré l'ambiguïté quasi omniprésente dans ses conclusions. Qu'il soit dirigé contre les bourgeois, les artistes ou les femmes, le dédain villiérien, à n'en pas douter, définit l'homme et l'œuvre. Dans l'univers villiérien, le mépris est une arme à double tranchant : il caractérise le regard des âmes nobles sur les serviteurs du sens commun mais, en même temps, se retrouve dans l'attitude des bourgeois matérialistes à l'égard de ces mêmes âmes. Conçu comme un fondement de l'esthétique qui vise l'idéal tout en luttant contre le réel positif, il joue un rôle important au niveau de la psychologie des personnages ce que reflètent leurs paroles, émotions et gestes.

Il n'en est pas moins vrai que le dédain ironique de Villiers reste inséparable de la notion de gloire à laquelle aspire cet écrivain méconnu par ses contemporains. Villiers conservait en lui les traits de ses ancêtres victorieux, ce dont témoigne son écriture même si sa modernité n'est pas à nier. C'était une attitude hautaine d'un aristocrate de sang et d'esprit qui adresse un appel idéaliste à des happy few.

${ }^{70}$ Cf. S. Tomkins, op. cit., p. 169.

${ }^{71}$ A. Villiers de l'Isle-Adam, L'Ève future, op. cit., p. 885.

${ }^{72}$ Idem, L'Inconnue, in CEuvres complètes, op. cit., t. I, p. 719-720.

${ }^{73}$ Ibid., p. 719. 
Aussi, le rapprochement que Remy de Gourmont instaure entre lui et Chateaubriand paraît-il justifié : « tous deux [...] recréèrent pour un temps l'âme de l'élite : de l'un naquit le catholicisme romantique et ce respect des traditionnelles vieilles pierres ; et de l'autre, le rêve idéaliste et ce culte de l'antique beauté intérieure $»^{74}$. Ce rêve a été forcé par l'écriture dans « une opération violente, artificielle, malaisée $»^{75}$ à l'aide d'un instrument tranchant et efficace, qui était celui de mépris.

\section{Bibliographie}

Bourre, Jean-Paul, Villiers de l'Isle-Adam. Splendeur et misère, Paris, Les Belles Lettres, 2002

Conrad, Thomas, « Le laboratoire de Villiers de l'Isle-Adam : manières d'écrire l'idéal », p. 1-11; in Ce qu'idéal veut dire : définitions et usages de l'idéalisme au XIX siècle, éd. Capucine Echiffre et al., 2015 ; URL : http://etudes-romantiques.ish-lyon.cnrs.fr/idealisme.html ; consulté le 8.01.2019

Correspondance générale de Villiers de l'Isle-Adam, éd. Joseph Bollery, Paris, Mercure de France, 1962 , t. I

Decottignies, Jean, Villiers le taciturne, Lille, Presses Universitaires de Lille, 1983

Dufief, Anne-Simone, "'Et c'était un crescendo de silences ». Villiers de 1'Isle-Adam », in Silences fin-de-siècle. Hommage à Jean de Palacio, éd. André Guyaux, Paris, PUPS, 2008, p. 167-179

Engelking, Ryszard, „Marzyciel szyderca” [« Rêveur railleur »], Literatura na świecie, 2001, n 1, p. 273-286

Glaudes, Pierre et Vibert, Bertrand, « Le théâtre du demi-monde dans les contes cruels », Littérature, 2014, no 71 , p. 197-228

Gourmont, Remy de, Le Livre des masques, Paris, Mercure de France, 1921

Gourmont, Remy de, Sixtine. Roman de la vie cérébrale, Paris, Union Générale d'Éditions, 1982

Gourmont, Remy de, «Un carnet de notes sur Villiers de l'Isle-Adam », L'Ermitage, 15 avril 1906, p. 223-238

Grand dictionnaire universel du XIX siècle, éd. Pierre Larousse, Paris, 1870, vol. VI ; 1874, vol. XI

Le Feuvre, Anne, Une Poétique de la récitation : Villiers de l'Isle-Adam, Paris, Honoré Champion, 1999

Liébart, Landry, «Un exemple d'intersection entre littérature et sociologie : le rapport entre l'écrivain et le journaliste au XIX ${ }^{\mathrm{e}}$ siècle », Equinoxes, printemps/été 2009, nº 12, n.p. ; URL : https://www. brown.edu/Research/Equinoxes/journal/Issue\%2012/eqx12_Liebart.html; consulté le 8.01.2019

Mallarmé, Stéphane, Euvres complètes, éd. Bertrand Marchal, Paris, Gallimard, 2003, t. II

Meitinger, Serge, « Mallarmé, poète et histrion », Romantisme, 1987, nº 55, p. 91-102

Noiray, Jacques, « La littérature comme vengeance : l'esthétique de Villiers de l'Isle-Adam d'après sa correspondance "», in L'Esthétique dans les correspondances d'écrivains et de musiciens (XIX $X^{e}-X X^{e}$ siècles), sous la dir. d'Arlette Michel, Paris, PUPS, 2001, p. $45-56$

Tomkins, Silvan, „Wstyd-upokorzenie a pogarda-wstręt: natura reakcji” [« Honte-humiliation et mépris-répulsion : la nature des réactions »], trad. Borys Szumański et Weronika Szwebs, Teksty Drugie, 2016, nº 4, p. 163-173

Verlaine, Paul, Euvres en prose complètes, éd. Jacques Borel, Paris, Gallimard, 1972

Vibert, Bertrand, «Conte cruel, conte cynique. Villiers, Mirbeau », in Cynismes littéraires, Pierre Glaudes, Jean-François Louette éd, Paris, Classiques Garnier, 2018, p. 147-163

\footnotetext{
${ }^{74}$ R. de Gourmont, Le Livre des masques, Mercure de France, 1921, p. 89-90.

75 Th. Conrad, op. cit., p. 1.
} 
Villiers de l'Isle-Adam, Auguste, Euvres complètes, éd. Alan Raitt et Pierre-Georges Castex, Paris, Gallimard, 1986, t. I et II

Watt Smith, Tiffany, Księga ludzkich uczuć [Le Livre des sentiments humains], trad. Jacek Konieczny, Warszawa, Wydawnictwo WAB, 2015

Anna Opiela-Mrozik, docteur ès lettres, maître de conférences à l'Institut d'Études Romanes de l'Université de Varsovie. Auteure du livre La Musique dans la pensée et dans l'œuvre de Stendhal et de Nerval (Paris, Honoré Champion, 2015) et d'articles consacrés aux relations entre la littérature et d'autres arts, en particulier la musique.

\begin{tabular}{|l|l|}
\hline CC) Comptive & $\begin{array}{l}\text { C by the author, licensee Łódź University - Łódź University Press, } \\
\text { Łódź, Poland. This article is an open access article distributed under } \\
\text { the terms and conditions of the Creative Commons Attribution license } \\
\text { CC-BY-NC-ND 4.0 (https://creativecommons.org/licenses/by-nc-nd/4.0/) }\end{array}$ \\
\cline { 2 - 2 } & Received: 2019-01-22; Accepted: 2020-12-08 \\
\hline
\end{tabular}

